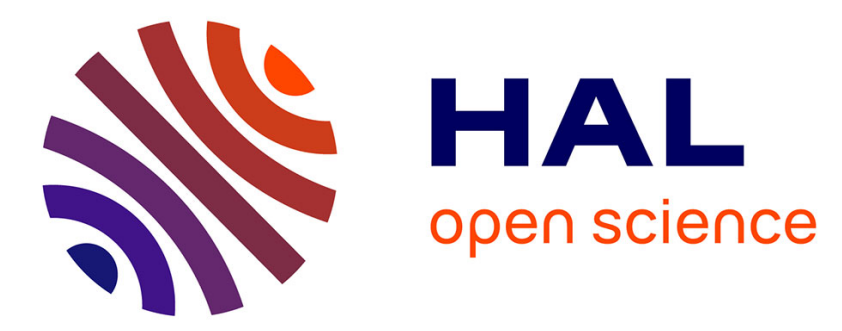

\title{
Dynamical effects in electron spectroscopy
}

Jianqiang Sky Zhou, J. J. Kas, Lorenzo Sponza, Igor Reshetnyak, Matteo Guzzo, Christine Giorgetti, Matteo Gatti, Francesco Sottile, J. Rehr, Lucia Reining

\section{- To cite this version:}

Jianqiang Sky Zhou, J. J. Kas, Lorenzo Sponza, Igor Reshetnyak, Matteo Guzzo, et al.. Dynamical effects in electron spectroscopy. Journal of Chemical Physics, 2015, 143 (18), pp.184109. 10.1063/1.4934965 . hal-02264126

\section{HAL Id: hal-02264126 https://hal.science/hal-02264126}

Submitted on 17 Nov 2021

HAL is a multi-disciplinary open access archive for the deposit and dissemination of scientific research documents, whether they are published or not. The documents may come from teaching and research institutions in France or abroad, or from public or private research centers.
L'archive ouverte pluridisciplinaire HAL, est destinée au dépôt et à la diffusion de documents scientifiques de niveau recherche, publiés ou non, émanant des établissements d'enseignement et de recherche français ou étrangers, des laboratoires publics ou privés. 


\section{AlP $\mid \begin{aligned} & \text { The Journal of } \\ & \text { Chemical Physics }\end{aligned}$}

\section{Dynamical effects in electron spectroscopy}

Jianqiang Sky Zhou, J. J. Kas, Lorenzo Sponza, Igor Reshetnyak, Matteo Guzzo, Christine Giorgetti, Matteo Gatti, Francesco Sottile, J. J. Rehr, and Lucia Reining

Citation: The Journal of Chemical Physics 143, 184109 (2015); doi: 10.1063/1.4934965

View online: http://dx.doi.org/10.1063/1.4934965

View Table of Contents: http://scitation.aip.org/content/aip/journal/jcp/143/18?ver=pdfcov

Published by the AIP Publishing

\section{AIP $\left.\right|_{\text {APL Photonics }}$}

APL Photonics is pleased to announce Benjamin Eggleton as its Editor-in-Chief

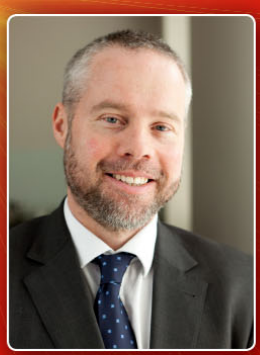




\title{
Dynamical effects in electron spectroscopy
}

\author{
Jianqiang Sky Zhou, ${ }^{1,2, a)}$ J. J. Kas, ${ }^{2,3}$ Lorenzo Sponza, ${ }^{4}$ Igor Reshetnyak, ${ }^{1,2}$ Matteo Guzzo, ${ }^{5}$ \\ Christine Giorgetti, ${ }^{1,2}$ Matteo Gatti, ${ }^{1,2,6}$ Francesco Sottile, ${ }^{1,2}$ J. J. Rehr, ${ }^{2,3}$ \\ and Lucia Reining ${ }^{1,2}$ \\ ${ }^{1}$ Laboratoire des Solides Irradiés, École Polytechnique, CNRS, CEA-DSM-IRAMIS, Université Paris-Saclay, \\ F-91128 Palaiseau, France \\ ${ }^{2}$ European Theoretical Spectroscopy Facility (ETSF) \\ ${ }^{3}$ Department of Physics, University of Washington, Seattle, Washington 98195-1560, USA \\ ${ }^{4}$ Department of Physics, King's College London, London WC2R 2LS, United Kingdom \\ ${ }^{5}$ Institut für Physik und IRIS Adlershof, Humboldt-Universität zu Berlin, D-12489 Berlin, Germany \\ ${ }^{6}$ Synchrotron SOLEIL, L'Orme des Merisiers, Saint-Aubin, BP 48, F-91192 Gif-sur-Yvette, France
}

(Received 12 June 2015; accepted 20 October 2015; published online 12 November 2015)

\begin{abstract}
One of the big challenges of theoretical condensed-matter physics is the description, understanding, and prediction of the effects of the Coulomb interaction on materials properties. In electronic spectra, the Coulomb interaction causes a renormalization of energies and change of spectral weight. Most importantly, it can lead to new structures, often called satellites. These can be linked to the coupling of excitations, also termed dynamical effects. State-of-the-art methods in the framework of many-body perturbation theory, in particular, the widely used GW approximation, often fail to describe satellite spectra. Instead, approaches based on a picture of electron-boson coupling such as the cumulant expansion are promising for the description of plasmon satellites. In this work, we give a unified derivation of the GW approximation and the cumulant expansion for the one-body Green's function. Using the example of bulk sodium, we compare the resulting spectral functions both in the valence and in the core region, and we discuss the dispersion of quasi-particles and satellites. We show that self-consistency is crucial to obtain meaningful results, in particular, at large binding energies. Very good agreement with experiment is obtained when the intrinsic spectral function is corrected for extrinsic and interference effects. Finally, we sketch how one can approach the problem in the case of the two-body Green's function, and we discuss the cancellation of various dynamical effects that occur in that case. @ 2015 AIP Publishing LLC. [http://dx.doi.org/10.1063/1.4934965]
\end{abstract}

\section{INTRODUCTION}

Density Functional Theory (DFT) ${ }^{1}$ has opened the way to a predictive first principles description of condensed-matter systems. Materials properties that are governed by the electronic ground state, such as crystal structures or cohesive energies, are often described within a precision of a few percent. In the Kohn-Sham $(\mathrm{KS})^{2}$ formulation an independentparticle Schrödinger equation is derived, and the resulting single-particle eigenfunctions allow one, in principle, to construct the exact density and total energy, and in practice, often to obtain very good approximations.

The KS equation also yields eigenvalues $\varepsilon_{i}^{0}$. However, besides the highest occupied state in a finite system these are not total energy differences of the system with $N$ and $N \pm 1$ particles, ${ }^{3,4}$ and they cannot be interpreted as electron addition and removal energies. The band structure given by the KS eigenvalues is therefore different from the one that is measured, e.g., in direct and inverse photoemission experiments, that is called the quasi-particle band structure, and in particular, the KS band gap is, in general, substantially smaller than the measured photoemission band gap (see, e.g., Ref. 5). This is sometimes called the KS band-gap problem.

\footnotetext{
a)jianqiang.zhou@polytechnique.edu
}

In order to go beyond the ground state and describe phenomena such as electron addition and removal, it is more appropriate to change the framework and work with Green's functions ${ }^{6,7}$ instead of the density. In particular, the imaginary part of the one-body Green's function yields the intrinsic spectral function that can be directly compared to direct and inverse photoemission spectra. In the independent-particle case, the spectral functions consist of peaks that are $\delta$-functions at the independent-particle energies. In an interacting system, the peaks are shifted and broadened: these are the quasiparticle peaks. Moreover, they lose weight towards additional structures and incoherent background that appear in the spectra, because all excitations are coupled, and the initial excitation decays by exciting electron-hole pairs, or collective excitations such as plasmons.

A route to approximate the one-body Green's function is traced by many-body perturbation theory (MBPT) ${ }^{6,7}$ In this framework, the one-body Green's function is determined from the Dyson equation $G=G^{0}+G^{0} \Sigma G$, where $G^{0}$ is the noninteracting Green's function and $\Sigma_{\sigma}\left(\mathbf{r}, \mathbf{r}^{\prime}, \omega\right)$ is the non-local and frequency-dependent self-energy that is diagonal in spin $\sigma$ when the Hamiltonian is not spin-dependent. If one is only interested in the position of the quasi-particle peaks, as it is most often the case, for example, if one only wants to calculate the quasi-particle band structure of a solid close to 
the Fermi level, it is sufficient to replace the local and static KS exchange-correlation potential $v_{\mathrm{xc}}$ in the KS equation by the real part of the exchange-correlation self-energy $\Sigma_{\mathrm{xc}}$ calculated at the real part $\varepsilon_{i}$ of the quasi-particle energy, i.e., $\operatorname{Re} \Sigma_{\mathrm{xc}}\left(\omega=\varepsilon_{i}\right){ }^{8,9}$ Here, we have defined $\Sigma=v_{H}+\Sigma_{\mathrm{xc}}$ where $v_{H}$ is the Hartree potential. The quasi-particle energy is the solution of the resulting Schrödinger-like equation, which requires, in principle, iterating the equation to determine $\varepsilon_{i}$. Since the self-energy is approximately linear around the quasi-particle energy, one can Taylor expand $\operatorname{Re} \Sigma_{\mathrm{xc}}\left(\omega=\varepsilon_{i}\right) \approx \operatorname{Re} \Sigma_{\mathrm{xc}}\left(\omega=\varepsilon_{i}^{0}\right)$ $+\left(\varepsilon_{i}-\varepsilon_{i}^{0}\right) \partial \operatorname{Re} \Sigma(\omega) / \partial \omega_{\mid \omega=\varepsilon_{i}^{0}}$. This leads to a renormalization of the quasi-particle corrections by a factor $Z \equiv[1-\partial \operatorname{Re} \Sigma(\omega) /$ $\left.\partial \omega_{\mid \omega=\varepsilon_{i}^{0}}\right]^{-1}$, called the quasi-particle renormalization factor. ${ }^{8-10}$

The non-locality in $\left(\mathbf{r}, \mathbf{r}^{\prime}\right)$ of the self-energy is tightly linked to the non-locality of the exchange in Hartree-Fock, for which Koopmans' theorem ${ }^{11}$ tells us that the eigenvalues have the meaning of electron addition and removal energies. In most cases, the fact that the self-energy is non-local is responsible for the opening of the band gap with respect to a KS calculation. The frequency-dependence of the selfenergy makes this object fundamentally different from any independent-particle potential. It causes the $Z$-factor to be smaller than one: $Z$ gives the fraction of spectral weight that remains in the quasi-particle peak, the rest being transferred to the satellites and the background. It is a measure of correlation, since in an independent-particle system (as in Hartree-Fock) it must be equal to one. Satellites can only appear when $Z$ is different from one, and they are always due to interactions.

The most widely used approximation to the self-energy is Hedin's $G W$ approximation. ${ }^{12}$ In this approximation, the self-energy is given as a convolution in frequency space of the one-body Green's function $G$ and the dynamically screened Coulomb interaction $W$, usually calculated in the random phase approximation (RPA). ${ }^{13}$ Since $G$ is the quantity to be calculated, the problem is, in principle, self-consistent. In practice, the self-energy is often constructed using a Green's function built with KS eigenvalues and eigenfunctions, both in $G$ and in $W$; the approach is then called $\mathrm{G}_{0} \mathrm{~W}_{0}$. Note that the exact measurable $W$, which is screened by the test-charge-testcharge dielectric function, can, in principle, be calculated using time-dependent density functional theory (TDDFT). The selfconsistent RPA $W$ is instead, in general, different from the measurable one. This aspect will be discussed later.

One of the early successes of the $\mathrm{G}_{0} \mathrm{~W}_{0}$ approximation was the solution of the "band-gap problem" for simple semiconductors and insulators, ${ }^{14,15}$ and since then, it has led to many successful band structure calculations in a wide range of materials ${ }^{8,9}$ Even energy levels in so-called strongly correlated materials like transition-metal oxides can be described (see, e.g., Refs. 16 and 17), as long as one stays in a low-temperature phase with magnetic ordering that leads to a unit cell with an even number of electrons. These materials, however, require an accurate description of localized $d$ - or $f$-electrons, which is often not given by simple KS functionals such as the Local Density Approximation (LDA), which have a tendency to delocalize charge. The problem can be overcome by carrying out the calculations self-consistently using some static approximation to the self-energy, such as the quasi-particle self-consistent GW (QSGW) approximation. ${ }^{5,18}$ This improves the density.
Also the fact that the eigenvalues of the KS solution can be far from the quasi-particle values can deteriorate results and updating quasi-particle eigenvalues in a self-consistent way is often useful to obtain better energies, further extending the range of materials for which the GWA is the method of choice for band structure calculations. ${ }^{19}$

However, as discussed above, quasi-particles are only a part of the measured spectra. The most interesting part when it comes to exploring correlation effects is contained in the satellites which directly exhibit the effects of coupling. Unfortunately the GWA turns out to have severe problems in describing satellites (see, e.g., Ref. 20). In principle, one expects that at least plasmon satellites are well described, since plasmons are the dominant structures that are seen in the inverse dielectric function $\epsilon^{-1}(\omega)$, and hence in the screened Coulomb interaction $W=\epsilon^{-1}(\omega) v_{c}$, where $v_{c}$ is the bare interaction. Satellites in X-ray spectra have an even longer history. Physically, these correlation effects arise from multi-electron excitations, e.g., shake-up and shake-off processes. ${ }^{21}$ In small molecules, these largely intrinsic processes can be treated to high accuracy, e.g., with configuration interaction methods. ${ }^{22}$ However, in condensed matter, additional collective excitations such as plasmons and phonons are present, which give rise to multiple satellites and strong dynamic correlation effects. Plasmons calculated in the RPA often compare well to experimental spectra such as electron energy loss spectra or inelastic x-ray scattering (see, e.g., Refs. 23-27). Satellites due to plasmons are indeed found in the GWA, but they are often too far from the quasi-particle energy, and sometimes much too sharp, and in other cases much too weak, as compared to experiment.

In the present work, we discuss why this is so, and how one can obtain a better description of satellite spectra. To this purpose, we derive the GWA from the fundamental functional differential equation that expresses the one-body Green's function as functional of an external potential, based on the approach of Schwinger. ${ }^{28}$ We highlight the two major approximations that lead to the GWA. Using the same set of equations we show how one can do better, ${ }^{29}$ deriving an exponential expression for $G$ that is equivalent to a cumulant representation. ${ }^{20}$ This is the topic of Section II. For an illustration, we show results for valence and core electron removal spectra of sodium as prototype examples. Section III gives the results of the GWA, with a particular accent on the importance of selfconsistency for the calculation of satellites. Cumulant results are contained in Section IV. In order to compare to experiment, the intrinsic spectral function is not sufficient: one has to add the extrinsic losses due to scattering of the outgoing electron on its way to the detector, as well as interference effects between extrinsic and intrinsic contributions. This is the topic of Section V. Finally, Section VI sketches a way to extend these ideas to the two-particle Green's function that would, in principle, treat the hole and the photoelectron on the same footing and therefore naturally contain intrinsic, extrinsic, and interference effects. Conclusions are given in Section VII.

\section{THEORETICAL FRAMEWORK}

The fundamental building block of MBPT is the onebody Green's function $G$. At zero temperature the time-ordered 
Green's function ${ }^{6}$ reads

$$
G(1,2) \equiv-i\left\langle\Psi_{0}\left|\mathbf{T}\left[\hat{\psi}(1) \hat{\psi}^{\dagger}(2)\right]\right| \Psi_{0}\right\rangle,
$$

where $\Psi_{0}$ is the many-body ground state, $\mathbf{T}$ is the timeordering operator, and $\hat{\psi}$ and $\hat{\psi}^{\dagger}$ are field operators in the Heisenberg picture. Here (1) stands for space, spin, and time coordinates $\left(\mathbf{x}_{1}, t_{1}\right) \equiv\left(\mathbf{r}_{1}, \sigma_{1}, t_{1}\right)$, and $\left(1^{+}\right) \equiv\left(\mathbf{x}_{1}, t_{1}^{+}\right)$with $t_{1}^{+}$ $\equiv t_{1}+\eta$ and $\eta \rightarrow 0^{+}$. The Green's function contains a wealth of information; in particular, the density is given by $n\left(\mathbf{x}_{1}\right)$ $=-i G\left(1,1^{+}\right)$and a diagonal element of the spectral function labelled $i$ in some basis is

$$
A_{i i}(\omega)=\frac{1}{\pi}\left|\operatorname{Im} G_{i i}(\omega)\right| .
$$

If the many-body ground state $\left|\Psi_{0}\right\rangle$ was known, one could evaluate the Green's function from Eq. (1). This is however, in general, not the case. Instead, one can use the fact that $G$ fulfills the equation of motion (note that $f(\overline{1}) g(\overline{1})$ stands for $\left.\int d 1 f(1) g(1)\right)$

$$
\begin{aligned}
G\left(1,1^{\prime}\right)= & G^{0}\left(1,1^{\prime}\right)+G^{0}(1, \overline{2}) v_{H}(\overline{2}) G\left(\overline{2}, 1^{\prime}\right) \\
& +i G^{0}(1, \overline{2}) v_{c}(\overline{2}, \overline{3}) L\left(\overline{2}, \overline{3}^{+}, 1^{\prime}, \overline{3}^{++}\right),
\end{aligned}
$$

where $G^{0}$ is the non-interacting Green's function, $v_{c}$ is the bare Coulomb interaction, $v_{H}$ is the Hartree potential, and $L$ is the two-particle correlation function that is linked to the two-body Green's function $G_{2}$ as $L=-G_{2}+G G$. However, also the two-particle Green's function $G_{2}$ is defined in terms of an expectation value of four field operators in the manybody ground state. Its equation of motion makes the threebody Green's function appear, and so on. In order to truncate this infinite chain and obtain a closed expression, one can use Schwinger's functional derivative approach ${ }^{28}$ that consists of introducing a fictitious external potential $u$ and expressing $L_{u}$ in the presence of $u$ as

$$
\frac{\delta G_{u}\left(2,1^{\prime}\right)}{\delta u(3)}=L_{u}\left(2,3,1^{\prime}, 3^{+}\right),
$$

where $G_{u}$ is $G$ for $u \neq 0$. This leads to the equation of motion in the compact form

$$
\begin{aligned}
G_{u}\left(1,1^{\prime}\right)= & G^{0}\left(1,1^{\prime}\right)+G^{0}(1, \overline{2}) \\
& \times\left\{\left[u(\overline{2})+v_{H u}(\overline{2})\right] G_{u}\left(\overline{2}, 1^{\prime}\right)+i v_{c}(\overline{2}, \overline{3}) \frac{\delta G_{u}\left(\overline{2}, 1^{\prime}\right)}{\delta u\left(\overline{3}^{+}\right)}\right\},
\end{aligned}
$$

where $v_{H u}$ is the Hartree potential built with the density $n_{u}(\mathbf{x})=-i G_{u}\left(\mathbf{x}, \mathbf{x}, t, t^{+}\right)$. If one could solve this functional differential equation, the solution at $u=0$ would be the desired one-body Green's function $G$. Note that when the last term is neglected, one retrieves the Hartree approximation for $G$. Instead, the variation of the Green's function goes beyond the independent-particle picture; it contains the information that excitations are coupled.

\section{A. MBPT in the linear-response approximation}

As pointed out in the book by Baym and Kadanoff, ${ }^{30}$ "there is no known technique to solve functional differential equations like [Eq. (5)] in an efficient way." Instead the equation can be used as a starting point for increasingly accurate approximations.

One of the complications of the equations is the fact that the density $n_{u}$ in the Hartree potential introduces a term that is quadratic in $G_{u}$. To overcome this problem, we introduce the total classical potential

$$
u_{\mathrm{cl}}(1)=u(1)+v_{H u}(1)
$$

and rewrite (5) as

$$
\begin{aligned}
G_{u}\left(1,1^{\prime}\right)= & G^{0}\left(1,1^{\prime}\right)+G^{0}(1, \overline{2}) u_{\mathrm{cl}}(\overline{2}) G_{u}\left(\overline{2}, 1^{\prime}\right) \\
& +i G^{0}(1, \overline{2}) v_{c}(\overline{2}, \overline{3}) \frac{\delta G_{u}\left(\overline{2}, 1^{\prime}\right)}{\delta u\left(\overline{3}^{+}\right)} \\
= & G^{0}\left(1,1^{\prime}\right)+G^{0}(1, \overline{2}) u_{\mathrm{cl}}(\overline{2}) G_{u}\left(\overline{2}, 1^{\prime}\right) \\
& +i G^{0}(1, \overline{2}) W_{u}(\overline{2}, \overline{3}) \frac{\delta G_{u}\left(\overline{2}, 1^{\prime}\right)}{\delta u_{\mathrm{cl}}\left(\overline{3}^{+}\right)}
\end{aligned}
$$

where we have defined the screened Coulomb interaction $W_{u}$ $=\epsilon_{u}^{-1} v_{c}$ with the time-ordered inverse dielectric function $\epsilon_{u}^{-1}$ $=\delta u_{\mathrm{cl}} / \delta u$.

This is still, in principle, exact, as long as all quantities depend on $u$, as indicated by the subscript. Note that $\epsilon_{u}^{-1}$ is not the usual linear response dielectric function, since it depends on the perturbing potential. However, since we are interested in the solution for vanishing $u$, a reasonable approximation is to evaluate the equation using $\epsilon_{u}^{-1} \approx \epsilon^{-1}$ at $u=0$. This corresponds to a linear-response approximation.

Written in a basis, the resulting equation reads

$$
\begin{aligned}
G_{i j}^{u}\left(t_{12}\right)= & G_{i j}^{0}\left(t_{12}\right)+G_{i m}^{0}\left(t_{13}\right) u_{\mathrm{cl}, m k}\left(t_{3}\right) G_{k j}^{u}\left(t_{32}\right) \\
& +i G_{i k}^{0}\left(t_{13}\right) W_{k l m n}\left(t_{34}\right) \frac{\partial G_{n j}^{u}\left(t_{32}\right)}{\partial u_{\mathrm{cl}, l m}\left(t_{4}\right)},
\end{aligned}
$$

where $t_{12} \equiv\left(t_{1}, t_{2}\right)$ or $\left(t_{1}-t_{2}\right)$ in equilibrium, $W_{k l m n}$ is a matrix element of the screened Coulomb interaction $W$, and we have replaced functional derivatives by partial derivatives, supposing the basis to be discrete, which corresponds to calculations in practice. Repeated indices are summed over. Note that we keep the symbol $G^{u}$, but with the understanding that now $G^{u}$ is a functional of $u_{\mathrm{cl}}$ instead of $u$.

\section{B. The GW approximation}

The simplest approximation to this differential equation is

$$
\frac{\partial G_{n j}^{u}\left(t_{32}\right)}{\partial u_{\mathrm{c}, l m}\left(t_{4}\right)} \approx G_{n l}\left(t_{34}\right) G_{m j}\left(t_{42}\right) .
$$

At $u=0$, this yields the Dyson equation $G$ $=G^{0}+G^{0} \Sigma^{G W} G$ with the GWA for the self-energy,

$$
\Sigma_{i m}^{G W}\left(t_{34}\right)=v_{H, i m}+i G_{n l}\left(t_{34}\right) W_{i l m n}\left(t_{34}\right) .
$$

The GWA involves hence two approximations: a linearization of the density response of the system to the perturbation given by the electron addition or removal and an approximation of the coupling of excitations.

Often the GWA is used with two further approximations: first, the Green's function $G$ that appears in Eq. (10) is replaced by an independent-particle one, for example, a Kohn-Sham 
Green's function. Second, $W$ is calculated in the RPA. As our derivation shows, these are additional approximations, not indispensable for the GWA. Instead, the derivation suggests that, in principle, $W$ should be the exact linear response screened Coulomb interaction, which is the time-ordered counterpart of the measurable retarded screened interaction (often called test-charge-test-charge interaction), and that the selfenergy should be built with the self-consistent Green's function $G$. As we will see later, the excitations contained in $W$ directly show up in the satellites. This allows one to confirm that, in agreement with our derivation, $W$ should indeed be the physical screened interaction, and not its RPA version.

\section{Beyond the GWA: Cumulant expression for the Green's function}

In order to go beyond approximation (9), we first introduce a quasi-particle Green's function $G^{Q P, u}$, defined as

$$
\left[G^{Q P, u}\right]_{i j}^{-1}=\left[G_{0}^{-1}\right]_{i j}-\left[u_{\mathrm{cl}}\right]_{i j}-\Sigma_{i j}^{G W}\left(\frac{\varepsilon_{i}+\varepsilon_{j}}{2}\right) .
$$

This allows us to rewrite Eq. (8) as

$$
\begin{aligned}
G_{i j}^{u}\left(t_{12}\right)= & G_{i j}^{Q P, u}\left(t_{12}\right)+i G_{i k}^{Q P, u}\left(t_{13}\right) W_{k l m n}\left(t_{34}\right) \frac{\partial G_{n j}^{u}\left(t_{32}\right)}{\partial\left[u_{\mathrm{c} 1}\right]_{l m}\left(t_{4}\right)} \\
& -G_{i k}^{Q P, u}\left(t_{13}\right) \Sigma_{k l}^{G W}\left(\frac{\varepsilon_{k}+\varepsilon_{l}}{2}\right) G_{l j}^{u}\left(t_{32}\right) .
\end{aligned}
$$

Following an idea of Ref. 29 we now approximately decouple the equations, by supposing that $G^{u}$ and $G^{Q P, u}$ are diagonal in the same, $u$-independent, basis. If one iterates Eq. (12) for $G_{i i}^{u}$ using this assumption, it turns out that $G_{i i}^{u}$ depends only on the element $\left[u_{\mathrm{cl}}\right]_{i i}$, which means that the screened interaction contributes only through the element

$\mathcal{W}\left(t_{34}\right) \equiv W_{i i i i}=\int d \mathbf{r} d \mathbf{r}^{\prime}\left|\phi_{i}(\mathbf{r})\right|^{2}\left|\phi_{i}\left(\mathbf{r}^{\prime}\right)\right|^{2} W\left(\mathbf{r}, \mathbf{r}^{\prime}, t_{34}\right)$,

where $\phi$ is the single-particle basis function labelled $i$. The resulting scalar differential equation for each matrix element $\mathcal{G}^{u} \equiv G_{i i}^{u}$ reads

$$
\begin{aligned}
\mathcal{G}^{u}\left(t_{12}\right)= & \mathcal{G}_{Q P}^{u}\left(t_{12}\right)+i \mathcal{G}_{Q P}^{u}\left(t_{13}\right) \mathcal{W}\left(t_{34}\right) \frac{\partial \mathcal{G}^{u}\left(t_{32}\right)}{\partial u_{\mathrm{cl}}\left(t_{4}\right)} \\
& -\mathcal{G}_{Q P}^{u}\left(t_{13}\right) \Sigma_{i i}^{G W}\left(\varepsilon_{i}\right) \mathcal{G}^{u}\left(t_{32}\right),
\end{aligned}
$$

where $u_{\mathrm{cl}} \equiv \int d \mathbf{r} u_{\mathrm{cl}}(\mathbf{r})\left|\phi_{i}(\mathbf{r})\right|^{2}$ is the diagonal element of the potential. Note that after decoupling, only the diagonals of $W$ and $\Sigma^{G W}$ are needed.

Eq. (14) can be solved exactly. Since it is a first order differential equation, it has more than one solution. Excluding a phase transition, the physical solution is the one that connects to the non-interacting one when $v_{c} \rightarrow 0$. This solution describes one orbital propagating in the medium given by $\mathcal{W}$, which represents the effect of all other electrons.

For $u \rightarrow 0$ the solution of (14) reads

$$
\begin{aligned}
\mathcal{G}\left(t_{12}\right)= & \mathcal{G}_{Q P}^{0}\left(t_{12}\right) e^{i\left(t_{1}-t_{2}\right) \Sigma_{i i}^{G W}\left(\varepsilon_{i}\right)} \\
& \times \exp \left[-i \int_{t_{1}}^{t_{2}} d t^{\prime} \int_{t^{\prime}}^{t_{2}} d t^{\prime \prime} \mathcal{W}\left(t^{\prime}-t^{\prime \prime}\right)\right] .
\end{aligned}
$$

The double integral of $\mathcal{W}$ leads to three terms,

$$
\begin{aligned}
-i \int_{t_{1}}^{t_{2}} d t^{\prime} \int_{t^{\prime}}^{t_{2}} d t^{\prime \prime} \mathcal{W}\left(t^{\prime}-t^{\prime \prime}\right) \\
=-\left(t_{1}-t_{2}\right) \frac{1}{2 \pi} \int d \omega \frac{\mathcal{W}(\omega)}{\omega} \\
\quad+\frac{i}{2 \pi} \int d \omega \frac{W(\omega)}{\omega^{2}}\left(e^{-i \omega\left(t_{1}-t_{2}\right)}-1\right),
\end{aligned}
$$

where the first term induces a shift of the quasi-particle peak, the second term leads to additional structures (the satellites), and the last term, which does not depend on the time difference, yields a renormalization of the spectrum. The decoupling approximation, although quite severe, allows us thus to elucidate the structure of the problem, and to highlight the physics that emerges from the solution of the linearized equation.

Let us first examine the term proportional to $\left(t_{1}-t_{2}\right)$ in Eq. (16), by comparing it to a GW quasi-particle shift. In the decoupling approximation, $\Sigma_{k k}^{G W} \approx i G_{k k} W_{k k k k}$. Evaluated at the quasi-particle energy, this yields exactly the term we are interested in. This means that $e^{-\left(t_{1}-t_{2}\right) \frac{1}{2 \pi} \int d \omega \frac{\mathcal{W}(\omega)}{\omega}}$ approximately cancels with the GW shift in $e^{i\left(t_{1}-t_{2}\right) \Sigma_{i i}^{G W}\left(\varepsilon_{i}\right)}$, and we are left with

$$
\mathcal{G}\left(t_{12}\right)=\mathcal{G}_{Q P}^{0}\left(t_{12}\right) \exp \left[\frac{i}{2 \pi} \int d \omega \frac{\mathcal{W}(\omega)}{\omega^{2}}\left(e^{i \omega\left(t_{1}-t_{2}\right)}-1\right)\right] .
$$

If one expands the exponential of the second term of Eq. (16), and by using the Kramers-Kronig relation between $\mathcal{W}$ and its imaginary part, one can see that this term makes a series of satellites appear on the high-binding energy side of the quasi-particle peak, since the imaginary part of $W$ contains the neutral excitations of the system. The satellite series corresponds to single, double, and multiple excitations. Such an expression is the exact solution ${ }^{32}$ of a core electron Hamiltonian, where a single fermion is coupled to bosons. ${ }^{32-36}$ Here, the bosons are the excitations contained in $\mathcal{W}$, and the fermion is given by $\mathcal{G}$. In the case of a core level, it is reasonable to divide the system into an isolated level and the rest, which screens the level. Here, we are also interested in spectra of valence electrons, where no such clear separation exists. However, one can still imagine that one excites a quasiparticle and that excitation of this quasi-particle, in turn, leads to bosonic excitations in the system. The quasi-particle is not simply the bare level; it is dressed by the other electrons. This is expressed by Eq. (17).

As above in the case of the quasi-particle shift, the description of the satellites can be improved by comparing the exponent to the GW self-energy in the decoupling approximation. One can see that the peaks on the high-binding energy side of the quasi-particle peak are then created by the imaginary part of $\Sigma^{G W}\left(\varepsilon_{i}-\omega\right)$ for $\mu>\varepsilon_{i}-\omega$, where $\mu$ is the chemical potential. Therefore, we replace $\operatorname{Im} \mathcal{W}(\omega)$ by $\operatorname{Im} \Sigma_{G W}^{h}$ $=\operatorname{Im} \Sigma^{G W}\left(\varepsilon_{i}-\omega\right) \theta\left(\mu-\varepsilon_{i}+\omega\right)$ in the first contribution of Eq. (17).

The last term in Eq. (17) is also evaluated using $\Sigma_{G W}^{h}$ such that the correct normalization of the spectral function is 
obtained. This leads to the final expression

$$
\begin{aligned}
\mathcal{G}\left(t_{12}\right)= & \mathcal{G}_{Q P}^{0}\left(t_{12}\right) \exp \left[\left.\frac{\partial \Sigma_{G W}^{h}(\omega)}{\partial \omega}\right|_{\omega=\varepsilon_{i}}\right] \\
& \times \exp \left[\frac{1}{\pi} \int_{\varepsilon_{i}-\mu}^{\infty} d \omega \frac{\operatorname{Im} \Sigma^{G W}\left(\varepsilon_{i}-\omega\right)}{(\omega-i \eta)^{2}} e^{(i \omega+\eta)\left(t_{1}-t_{2}\right)}\right] .
\end{aligned}
$$

This is the time-ordered cumulant (TOC) approximation for the hole part of the Green's function. Here we are interested in photoemission where $i$ stands for an occupied state, but the same approach can be used to describe inverse photoemission spectra. ${ }^{31}$ In both cases, the electron addition and removal sectors are decoupled by the approximation.

Several derivations of this or closely related expressions can be found in the literature. In particular, Langreth ${ }^{32}$ has obtained the cumulant Green's function as a solution to an electron-boson coupling Hamiltonian. In the simplest case of one level and one boson, such a Hamiltonian corresponds to Eq. (14), where $\mathcal{W}$ contains one bosonic excitation. The more complete equation (8) couples several fermionic levels and bosonic excitations. With respect to Langreth, our derivation starts from the full many-body Hamiltonian, and not from the already approximate electron-boson coupling model. The solution of the problem is then based on a different mathematical strategy, but of course with similar results, since in both cases the solution is the exact solution to the approximate problem. Other derivations postulate the GWA self-energy as the central ingredient, and either solve the equation of motion for the Green's function approximately, ${ }^{37}$ or use the cumulant exponential form as ansatz, with the cumulant derived from the constraint that the Dyson equation and the cumulant approach should yield the same result to first order in $W .{ }^{38}$ In our case, the GWA form of the self-energy appears naturally. The closest derivation to ours is probably the one of Hedin, ${ }^{39}$ where the diagrammatic expansion of the Green's function is resummed, thanks to approximations on the recoil effects. As in our derivation, there is no model Hamiltonian, $W$ is the central ingredient, and the GWA self-energy appears as a result.

Eq. (17) or (18) in several variants has been used in $a b$ initio calculations to describe photoemission spectra of various metals and semiconductors. ${ }^{31,38,40-44}$ In Refs. 20, 45, and 46, Eq. (18) was applied to silicon and graphite. By including also extrinsic and interference effects (see Sec. V), this led to results in excellent agreement with experiment. Here, we follow this route. One can also use the method outlined above to go beyond and derive more sophisticated approximations such as the retarded cumulant approximation; ${ }^{47}$ this is however not the topic of the present work.

Because of the direct link between the GWA and the TOC, cumulant results are obtained at almost no additional cost once a GWA calculation has been performed. In particular, one can calculate $\operatorname{Im} \Sigma^{G W}\left(\varepsilon_{i}-\omega\right)$ and make a fit using a many-pole representation with weights $a$ and pole frequencies $\tilde{\omega}$. The general many-pole expression gives, in principle, an exact representation of $\operatorname{Im} \Sigma^{G W}\left(\varepsilon_{i}-\omega\right) \cdot{ }^{45,46,48}$ For clarity of the discussion, we give here the resulting spectral function $A_{i}(\omega)=1 / \pi|\operatorname{Im} \mathcal{G}(\omega)|$ for the case of a single pole,

$$
A_{i}(\omega)=\frac{e^{-a_{i}}}{\pi} \sum_{n=0}^{\infty} \frac{a_{i}^{n}}{n !} \frac{\Gamma_{i}}{\left(\omega-\varepsilon_{i}+n \tilde{\omega}_{i}\right)^{2}+\Gamma_{i}^{2}},
$$

where $\varepsilon_{i}$ and $\Gamma_{i}$ are real and imaginary parts of the QP energy, respectively. The spectral function $A_{i}(\omega)$ shows the quasiparticle peak at $\varepsilon_{i}$, followed by a Poisson-like decay of satellite replicas at energies $\varepsilon_{i}-n \tilde{\omega}_{i}$. Numerical results shown below are obtained with many poles, typically a few hundred. Note that in silicon ${ }^{20}$ a single plasmon-pole model was sufficient to obtain good results, but a metal close to the Fermi level requires a much finer frequency sampling.

\section{Calculations in practice}

In order to illustrate the theory, we present results for the electron removal spectrum of bulk sodium obtained with ab initio calculations using a plane wave basis and pseudopotentials as implemented in the ABINIT code. ${ }^{49}$ This simple metal has been extensively studied and, in particular, it has been shown ${ }^{38,50}$ that the GWA does not yield the experimentally observed plasmon satellite series, whereas the cumulant approximation does. ${ }^{38}$ However, these studies were limited to the valence band, and even there, a detailed analysis is still missing. Here, we provide this analysis, pointing out the role of a spurious plasmaron in the bad performance of the GWA. Moreover, we include the $\mathrm{Na} 2 \mathrm{~s}$ and $2 \mathrm{p}$ core in the calculation, thus treating valence and core on the same footing.

Of particular interest is the question of self-consistency. The quasi-particle energies that appear in Eq. (18) should, in principle, be calculated self-consistently. However, this requires several sometimes cumbersome calculations, and in many materials it is a good approximation to build the selfenergy using the results of simpler calculations, such as a Kohn-Sham band structure. In the framework of the GWA, this way to determine the quasi-particle energy $\varepsilon_{i}$ is called a $\mathrm{G}_{0} \mathrm{~W}_{0}$ calculation. Through this approximation the KohnSham band structure determines then also the cumulant result. In simple metals, the LDA is often the approximation of choice. However, as Sec. III will show, self-consistency is a critical issue when moving to the core levels, even in simple metals where the valence band is almost not affected. The most important contribution to self-consistency comes from the energies. Therefore we also perform calculations where the energies in $G$ are updated until convergence, while $W_{0}$ is kept fix, since it is supposed to approximate the measurable screened interaction. This is called energy self-consistent $\mathrm{GW}_{0}$ in the following.

Calculations have been performed for the experimental sodium crystal structure at temperature $\mathrm{T}=5 \mathrm{~K} .{ }^{51}$ The lattice constants are taken to be $4.225 \AA$. We have generated a sodium Troullier-Martins-type pseudopotential ${ }^{52}$ with semicore and valence states $(2 \mathrm{~s}, 2 \mathrm{p}$, and $3 \mathrm{~s}) .^{53}$ The Brillouin zone of sodium was sampled using a $16 \times 16 \times 16$ grid mesh that yields 145 inequivalent k-points in the irreducible Brillouin zone (BZ), and a smearing temperature of $0.01 \mathrm{Ha}$ was used. This is a fictitious temperature that only serves as a computational trick to speed up the k-point convergence, which explains why we can still use a standard time-ordered formalism. We have checked that our results are not biased by the value of the temperature. The plane-wave cutoff of the LDA ground-state calculation was 
200 hartree. We have used the same RPA screening $W_{0}$ for both one shot $\left(\mathrm{G}_{0} \mathrm{~W}_{0}\right)$ and energy-self-consistent $\left(\mathrm{GW}_{0}\right)$ calculation with 60 bands, 100 plane waves representing the wave functions and the dielectric matrix. Note that the update of the energies can be expected to be the most important contribution to self-consistency in sodium. The full frequency dependence of the self-energy was calculated using a contour-deformation technique. We calculated the screening using 100 frequencies on the real axis up to an energy of $25 \mathrm{eV}$ and 10 frequencies on the imaginary axis. The final self-energy was converged using 60 bands, 9000 plane waves for both wave functions and the exchange term. The cumulant expansion method and the extrinsic/interference technique are the ones used in Refs. 20 and 45. In particular, we evaluate Eq. (18).

\section{THEORETICAL PHOTOEMISSION SPECTRUM OF SODIUM: GWA RESULTS}

Before looking at the final results, it is useful to analyze the most important ingredient, namely, the GWA. In particular, we will compare results of $\mathrm{G}_{0} \mathrm{~W}_{0}$ based on the LDA, and energy self-consistent $\mathrm{GW}_{0}$.

\section{A. Quasiparticles and plasmon satellites in core and valence}

Fig. 1 shows the spectral function of the $3 \mathrm{~s}$ valence band at the $\Gamma$ point (lower panel). To understand the origin of the fea-

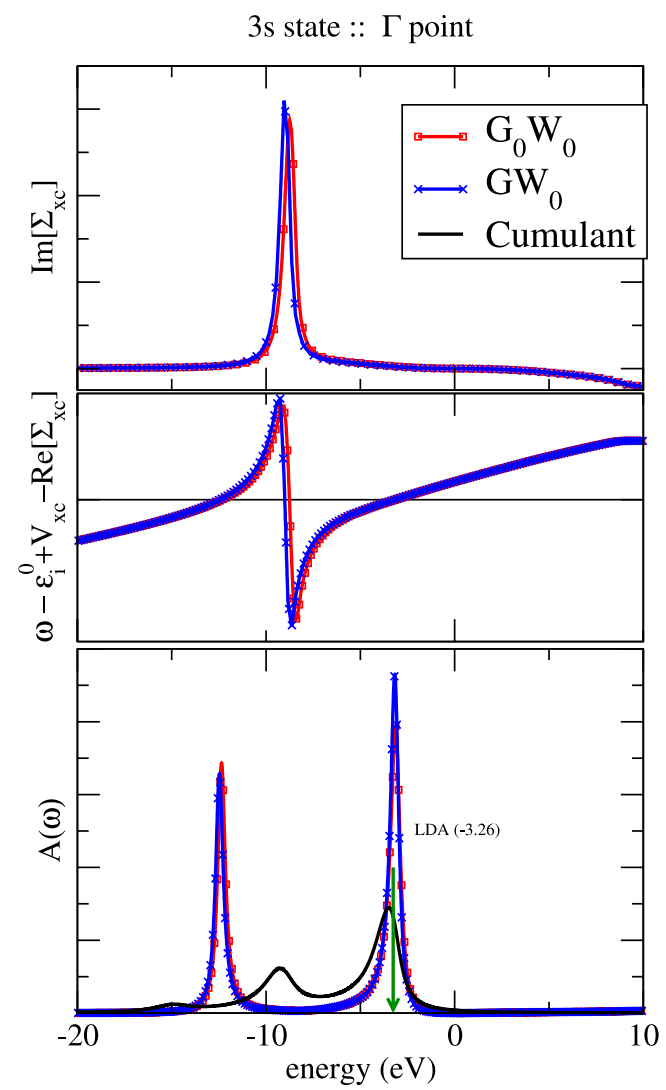

FIG. 1. Valence band intrinsic spectral function of sodium at the $\Gamma$ point (lower panel). Red line is $\mathrm{G}_{0} \mathrm{~W}_{0}$ results, blue line is $\mathrm{GW}_{0}$. Black line is the result of the cumulant expansion. The position of the LDA band at $\Gamma$ is given by the green arrow. The upper two panels show the imaginary and shifted real parts of the self-energy. The zero of the energy axis is set to the $\mathrm{GW}_{0}$ Fermi energy. tures, we also show the imaginary part of the self-energy (upper panel) as well as the shifted real part (note that in the figure $\varepsilon_{i}^{0}$ denotes a KS eigenvalue, therefore $\left(\omega-\varepsilon_{i}^{0}+V_{\mathrm{xc}}-\operatorname{Re} \Sigma_{\mathrm{xc}}(\omega)\right)$ is plotted to avoid double counting; middle panel). The quasiparticle condition is fulfilled when this function crosses zero. Red is $\mathrm{G}_{0} \mathrm{~W}_{0}$ results, blue is $\mathrm{GW}_{0}$ (i.e., updated energies in $G$ ). The imaginary part of $\Sigma$ shows one pronounced peak about $6 \mathrm{eV}$ below the quasi-particle energy, corresponding to the plasmon energy of sodium. In correspondence, the real part is strongly dispersing. It crosses zero at the quasi-particle energy, giving rise to the quasi-particle peak of the bottom valence band. It is close to the band energy found in the LDA, and there is just a slight band narrowing, as is also found in the homogeneous electron gas. However, there is also a second crossing, around $12 \mathrm{eV}$ binding energy. This causes a second pronounced peak in the spectral function. This is the so-called plasmaron. It is further away from the quasi-particle peak than the plasmon energy, which explains the bad agreement between $\mathrm{G}_{0} \mathrm{~W}_{0}$ and experiment found also in the earlier work. ${ }^{38}$ Self-consistency does not change the results significantly.

The results for the core levels are given in Fig. 2, with analogous plots as above. At the left is the $\mathrm{Na} 2 \mathrm{~s}$ level, at the right $\mathrm{Na} 2 \mathrm{p}$. Again the $\mathrm{G}_{0} \mathrm{~W}_{0}$ and $\mathrm{GW}_{0}$ satellites are of plasmaronic origin and therefore too sharp and too far away from the quasi-particle energy as compared to the plasmon frequency.

\section{B. Importance of self-consistency}

Two things should be noted in the core level spectra. First, $\mathrm{G}_{0} \mathrm{~W}_{0}$ and $\mathrm{GW}_{0}$ give very different results. This is due to the fact that the GWA shifts the core level significantly with respect to the LDA. Energy self-consistency is therefore mandatory for the core. Second, in the non-self-consistent calculation, the commonly made assumption that one could calculate quasi-particle levels from the linearized self-energy cannot be made. Indeed, the arrow labeled $\mathrm{G}_{0} \mathrm{~W}_{0}$ indicates the quasi-particle energy that one would obtain by using that procedure: clearly, the energy is completely off with respect to the quasi-particle peak in the $\mathrm{G}_{0} \mathrm{~W}_{0}$ spectral function (red curve). Moreover, in the non-self-consistent $\mathrm{G}_{0} \mathrm{~W}_{0}$ calculation the satellite is much farther from the quasi-particle peak and the weight transfer from the quasi-particle to the satellite is huge, which cannot be justified with any particular strong correlation effect in sodium. Indeed, the situation changes drastically when self-consistent calculations are performed, as one can see for the blue curves: the satellites get closer to the quasi-particle peak (they are located at a distance $\sim 1.5$ times the plasmon energy) and their intensity is reduced. It has been noted in several places that eigenvalue self-consistency is crucial when one is interested in GW satellites; ${ }^{19,31,54,55}$ the present example is a striking illustration. Overall, the two core levels behave similarly, although all features discussed here are more pronounced in the deeper core.

\section{Satellite dispersion}

The imaginary part of the self-energy is an integral over the plasmon spectrum. The small values of momentum transfer 

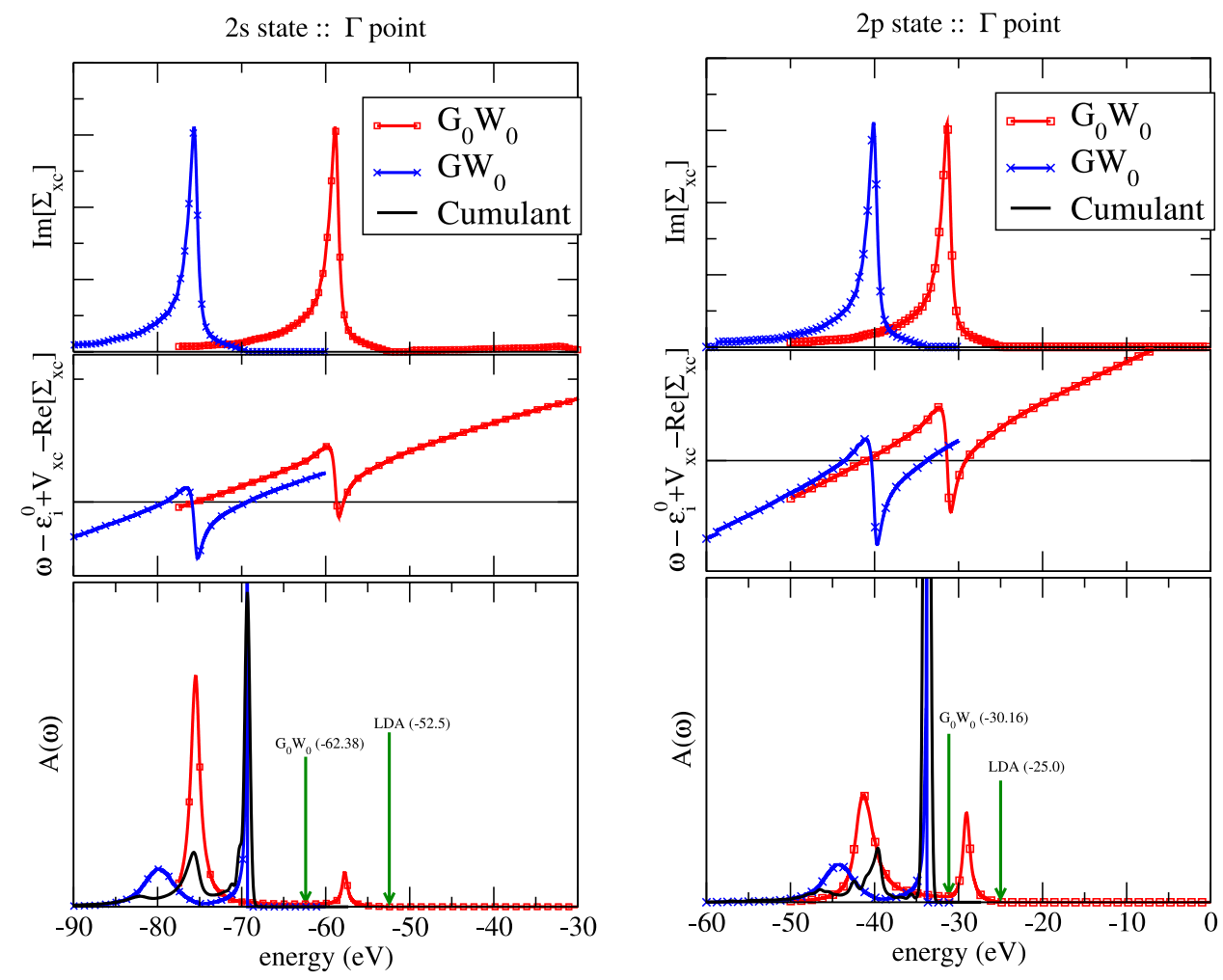

FIG. 2. Intrinsic spectral function of sodium at the $\Gamma$ point (lower panel) for the core levels $\mathrm{Na} 2 \mathrm{~s}$ (left) and $\mathrm{Na} 2 \mathrm{p}$ (right). Red is $\mathrm{G}_{0} \mathrm{~W}_{0}$ results, blue line is $\mathrm{GW}_{0}$. Black line is the result of the cumulant expansion. The position of the LDA band at $\Gamma$ is given by the green arrow labeled LDA. The arrow labeled $\mathrm{G}_{0} \mathrm{~W}_{0}$ indicates the quasi-particle energy that one would obtain by the usual first-order approximation based on a linearization of the self-energy. The upper two panels show the imaginary and shifted real parts of the self-energy.

dominate this integral, which explains why the imaginary part is relatively sharp. If the spectral function followed $\operatorname{Im} \Sigma$, the GWA satellite would disperse together with the valence band, at a distance about the plasmon energy. Fig. 3 shows the k-resolved valence band spectra (the core spectra do not disperse). GW results are on the left. At the $\Gamma$-point (bottom panel) the satellite corresponds to a sharp plasmaron peak, whereas it smoothens for k-points closer to the Fermi level. Still, the satellite peak position disperses and follows essentially the quasi-particle peak, although it has a slight tendency to get closer when the plasmaron effect becomes weaker, and the distance between quasi-particle and satellite is overestimated in the GWA.

\section{THEORETICAL PHOTOEMISSION SPECTRUM OF SODIUM: CUMULANT RESULTS}

Since the non-self-consistent $\mathrm{G}_{0} \mathrm{~W}_{0}$ results for the core are so bad, they are not suitable as input for the cumulant calculation, and we only show the final results that are obtained using
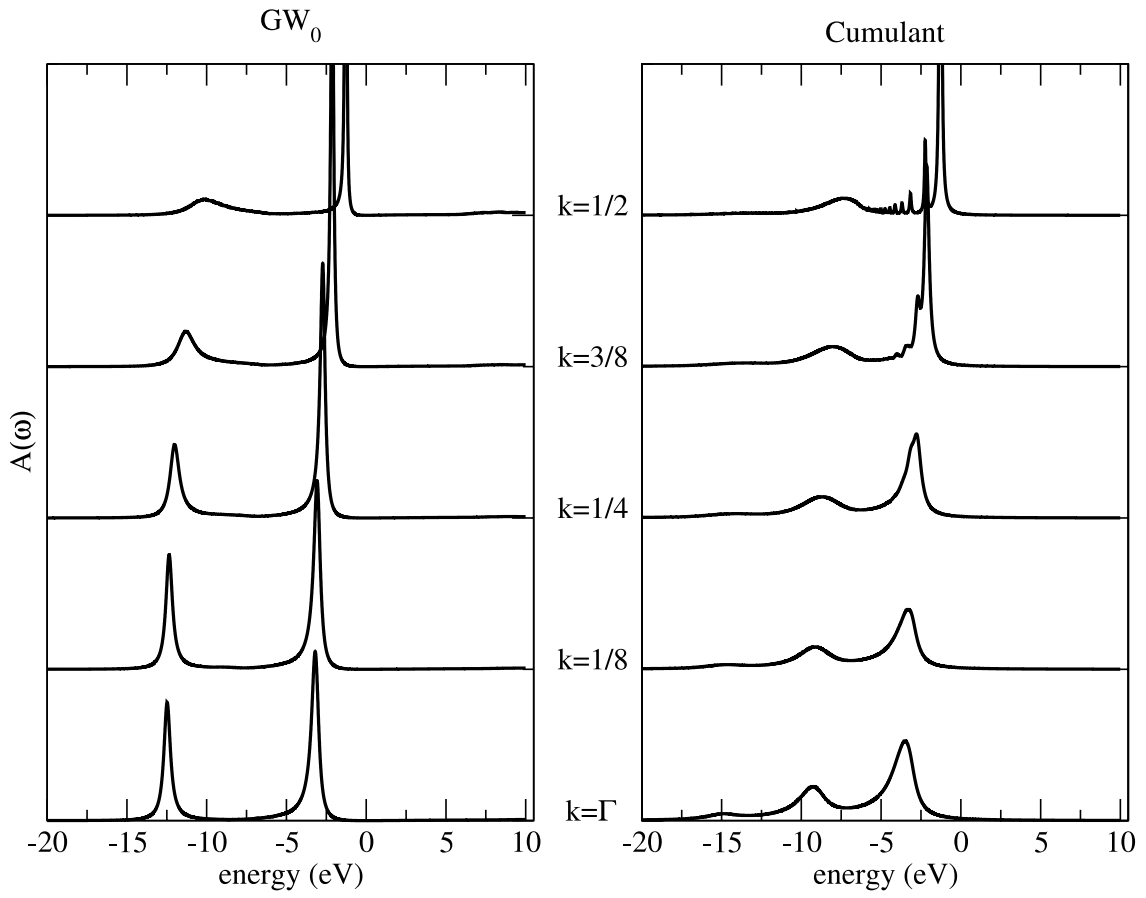

FIG. 3. k-resolved valence band spectral functions in sodium along $Г Н$ (k points are expressed in units of the $\Gamma \mathrm{H}$ length). Left panel: GWA results. Right panel: results of the cumulant expansion. 
as input the energy self-consistent $\mathrm{GW}_{0}$ self-energy. Here, we would like to remind that, according to our derivation, the screened Coulomb interaction should, in principle, be the timeordered version of the measurable test charge-test charge interaction that one could calculate, e.g., using TDDFT. The RPA based on Kohn-Sham ingredients is to be seen as a reasonable approximation, in many cases better than, e.g., RPA based on GW Green's functions. Therefore, we use $W_{0}$ calculated in LDA-RPA everywhere.

The black curves in Figs. 1 and 2 show the cumulant results. Note that in all cases two satellites can be resolved, at a distance of once and twice the calculated plasmon frequency, as expected. The first satellite is closer to the quasi-particle than the GW satellite, since the latter represents rather an average of the cumulant series.

The k-resolved cumulant spectral function is shown in the right panel of Fig. 3. The satellite clearly follows the quasiparticle dispersion. These results are similar to the results for the $\mathrm{HEG},{ }^{47}$ in particular, concerning the dispersion. The same behaviour has recently also been observed in silicon. ${ }^{43,44}$ However, comparison with experiment is not as crisp, as the plasmon replicas are blurred by dispersion and effects of extrinsic losses and interference as discussed below. The main difference to the spectra in Ref. 47 is the fact that in that work on the HEG the retarded cumulant method was applied, whereas here we use the original time-ordered version. Therefore, our spectral function does not have weight on both sides of the Fermi level, contrary to the GW one. However, the retarded cumulant and more sophisticated versions have other drawbacks and need more testing, which makes them less suitable for the purpose of the present paper, which is an analysis of the main effects and the discussion of core spectra. The core levels and valence states that are not very close to the Fermi level do not couple to the unoccupied space, so that TOC and retarded cumulant give the same results.

\section{TOWARDS EXPERIMENTAL SPECTRA: EXTRINSIC AND INTERFERENCE EFFECTS}

The observed result of a photoemission measurement is more than just an intrinsic spectral function. In principle, one has a complex process of excitation of two particles with their mutual interaction and interaction with all system particles, the electron travelling through the system and the surface to the detector, coupling to phonons, a secondary electron background, etc. (see, e.g., Ref. 56 for some discussion). It is desirable to treat the main effects at least approximately. Concerning the extrinsic losses of the travelling electron and the interference effects with the intrinsic excitation of electron-hole pairs and plasmons by the hole, the theory can be greatly simplified by making use of the quasi-boson approach of Hedin and Bardyszewski ${ }^{57}$ and Hedin, Michiels, and Inglesfield (HMI), ${ }^{58}$ for treating extrinsic losses. Of key importance is the treatment of valence excitations due to the interaction with the photoelectron (extrinsic excitations) and the hole (intrinsic excitations), as well as interference between these processes. The HMI model describes excitations of a semi-infinite jellium coupled to a hole as well as a photoelectron. The excitations of the jellium are bosonic in nature, i.e., plasmons and surface plasmons, etc. Thus, the model can be associated with a bosonic Hamiltonian, where the electron-boson coupling is related to the screened Coulomb interaction. The strength of the intrinsic excitations depends mostly on the coupling, and surface effects can be neglected, while the extrinsic excitations depend on the distance travelled by the photoelectron through the material, and thus on the inelastic mean free path. Within these approximations the photocurrent is given by

$$
\begin{aligned}
J_{k}\left(\omega_{0}\right)= & \sum_{i}\left|M_{i k_{0}}\right|^{2} \int_{0}^{\infty} d z_{h} e^{-a_{k}\left(z_{h}\right)} \int_{-\infty}^{\infty} d t e^{i\left(\omega_{0}-\epsilon_{k}+\epsilon_{i}\right) t} \\
& \times \exp \left[\int d \omega \gamma_{k}\left(\omega, z_{h}\right) e^{-i \omega t}\right] .
\end{aligned}
$$

Here, $\omega_{0}$ is the photon frequency, $k_{0}=\sqrt{2\left(\omega_{0}+\epsilon_{i}\right)}$ is the threshold photoelectron momentum, $M_{i k}$ are the dipole matrix elements between hole-state $|i\rangle$ and photoelectron $|\boldsymbol{k}\rangle$, and $\boldsymbol{k}$ and $\epsilon_{k}=k^{2} / 2$ denote the photoelectron momentum and kinetic energy at the detector. The amplitude reduction $a_{k}\left(z_{h}\right)$ for a hole located a distance $z_{h}$ from the surface is related to the inelastic mean free path $\lambda_{k}$ by

$$
a_{k}\left(z_{h}\right) \approx \int d \omega \gamma_{k}\left(\omega, z_{h}\right)=2 z_{h} / \lambda_{k}+a_{i n t}\left(z_{h}\right)
$$

In the above equation, we have made the approximation that the matrix elements $M_{i k}$ are constant over the range of photoelectron energies of interest, i.e., from threshold to several multiples of the plasmon frequency below threshold. The function $\gamma_{k}\left(\omega, z_{h}\right)$ is given as a sum over boson modes with momentum $\boldsymbol{q}$ and excitation energy $\omega_{\boldsymbol{q}}$, i.e.,

$$
\gamma_{\boldsymbol{k}}\left(\omega, z_{h}\right)=\sum_{\boldsymbol{q}}\left|g_{\boldsymbol{q}, \boldsymbol{k}}\left(\omega, z_{h}\right)\right|^{2} \delta\left(\omega-\omega_{q}\right)
$$

and can be interpreted as the single boson excitation spectrum. Assuming that the semi-infinite solid occupies the space $z<0$, the coupling is given by

$$
g_{\boldsymbol{q}, \boldsymbol{k}}\left(\omega, z_{h}\right)=\frac{V^{\boldsymbol{q}}\left(z_{h}\right)}{\omega}+\frac{i}{\kappa} \int_{-\infty}^{z_{\boldsymbol{h}}} e^{i(\tilde{k}-\kappa)\left(z-z_{h}\right)} V^{\boldsymbol{q}}(z) d z,
$$

where the electron-boson coupling functions (fluctuation potentials) $V^{q}$ are related to the screened Coulomb interaction. In our model calculations, we use the bulk and surface plasmon fluctuation potentials of Inglesfield. ${ }^{59}$ In the above equation, the first term couples to the hole, the second to the photoelectron, and the cross terms in $\left|g_{\boldsymbol{q}, \boldsymbol{k}}\left(\omega, z_{h}\right)\right|^{2}$ give the interference. Thus, the excitation spectrum can also be separated into intrinsic, extrinsic, and interference terms, i.e., $\gamma_{k}=\gamma^{i n t}+\gamma_{k}^{e x t}+\gamma_{k}^{i n f}$. We find that the intrinsic couplings (and thus amplitudes) are very weakly dependent on the distance from the surface $z_{h}$, and can thus be approximated by a constant. The wave numbers $\tilde{k}$ and $\kappa$ coincide with the time-inverted low-energy electron diffraction (LEED) state, ${ }^{60}$

$$
|\tilde{k}\rangle=e^{i \boldsymbol{K} \cdot R}\left[\theta(z) e^{-i \tilde{k}^{*} z}+\theta(-z) e^{-i \tilde{k} z}\right]
$$

and are given by

$$
\begin{aligned}
& \tilde{k}=\sqrt{k^{2}+2\left(\phi+\epsilon_{F}+i \Gamma\left(\epsilon_{k}\right)\right)}, \\
& \kappa=\sqrt{2\left(\omega_{0}+\phi+\epsilon_{F}+i \Gamma\left(\omega_{0}\right)\right)-|\boldsymbol{Q}+K|^{2}},
\end{aligned}
$$


where $\phi$ is the work function and $\epsilon_{F}$ is the Fermi energy. The bold capital letters indicate vectors parallel to the surface, e.g, $\boldsymbol{k}=\{\boldsymbol{K}, k\}$ and $\boldsymbol{q}=\{\boldsymbol{Q}, q\}$. Finally, $\Gamma$ is an imaginary energy related to the photoelectron lifetime inside the material and gives the photoelectron mean free path, $\lambda_{k}=1 / \operatorname{Im} \tilde{k}$. The dependence on the vector $\boldsymbol{K}$ gives the spectral dependence on the outgoing angle of the photoelectron. Thus, the model is a good way to estimate the amplitude of extrinsic and interference effects for varying photon energy and detection angle for semi-infinite solids.

The high kinetic energy photoelectron is approximated using a plane-wave time-inverted LEED state which is damped inside the solid by the inelastic mean free path and behaves like a plane wave far from the solid surface. This damping is extremely important, and without it, the current from a semi-infinite solid is unbounded since electrons at all depths contribute equally to the intrinsic signal. The use of a planewave inverted LEED state for high kinetic energy photoelectrons is a reasonable approximation; however, the localized approximation is less accurate for the valence hole. Consequently, we use this model only to calculate the weight of the extrinsic plus interference satellites and modify the amplitude of the intrinsic satellites calculated according to the more accurate theory outlined in Sec. II C. In particular, we scale the correlation part of the self-energy in Eq. (18) by the ratio of the total to intrinsic weights (averaged over $z_{h}$ ) as calculated using the HMI model for plasmon frequency $\omega^{\prime}$,

$$
R\left(\omega^{\prime}\right)=\frac{\bar{a}_{\mathrm{tot}}\left(\omega^{\prime}\right)}{\bar{a}_{\text {int }}\left(\omega^{\prime}\right)},
$$

where the averaged amplitudes are given by

$$
\begin{aligned}
& \bar{a}_{t o t}=\int d z_{h} d \omega e^{-a_{k}\left(z_{h}\right)} \gamma_{k}\left(\omega, z_{h}\right), \\
& \bar{a}_{\text {int }}=\int d z_{h} d \omega e^{-a_{k}\left(z_{h}\right)} \gamma_{i n t}\left(\omega, z_{h}\right),
\end{aligned}
$$

and the dependence on the plasmon frequency $\omega^{\prime}$ is implicit in the above equations. This modification of the amplitude is possible since the exponential forms of the two approaches are similar and allows for a good approximation of the total satellite weight. ${ }^{20}$ In order to compare to the experiment, the secondary electron background is also added to the spectral function. The background is approximately proportional to an integral of the spectral function (or sum of spectral functions in the case of the valence electrons), i.e., $b(\omega)=c \times \int_{\infty}^{\omega} d \omega^{\prime} A\left(\omega^{\prime}\right)$. Thus, the total calculated photoelectron current is given by $J_{k}(\omega)$ $=A(\omega)[1+b(\omega)]$, where the constant of proportionality is set by matching to the experimental data at low energy where the spectral function is small, and the background dominates the signal.

The final results for the photocurrent calculated including intrinsic, extrinsic, and interference effects are shown in Fig. 4 for the valence band, and in Fig. 5 for the core level. The calculation of the $2 \mathrm{~s}$ spectrum in Fig. 5 also includes an approximation for the surface plasmon contribution, which appears as a shoulder at an excitation energy of approximately $\omega_{p} / \sqrt{2}=4.25$. Our calculations are given by the red solid curve; the experimental photoemission spectra ${ }^{61,62}$ (measured at $1486.7 \mathrm{eV}$ photon energy) are shown in black dots. For

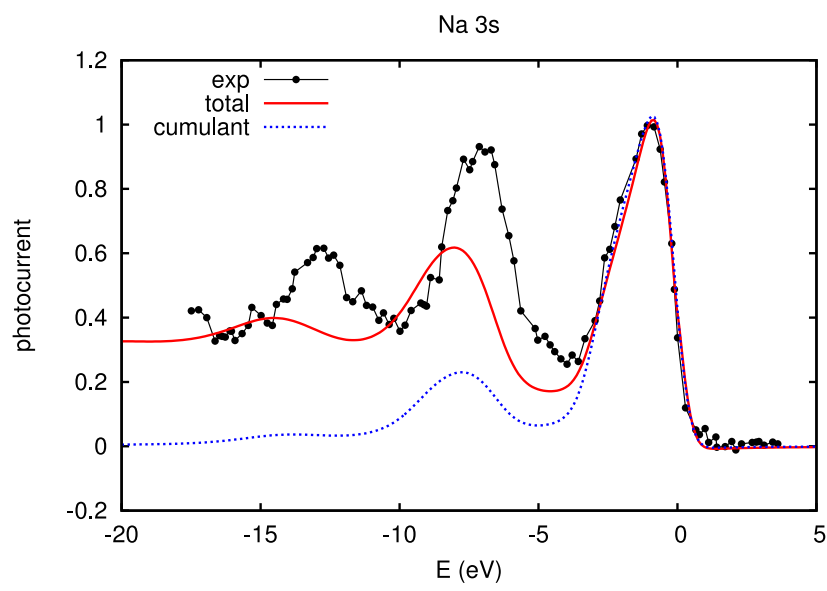

FIG. 4. Comparison of the experimental photoemission spectrum to the spectrum calculated using the cumulant + extrinsic + interference approach: valence band. Red solid line is total calculation, including a secondary electron background, black line with circles is experiment, ${ }^{61}$ and blue dashed line is the intrinsic cumulant spectral function. The spectra are angle-integrated. Original experimental data were in arbitrary units: here we have normalized them to the main-peak intensities.

comparison, the figures also include the momentum-integrated cumulant spectral function without extrinsic, interference, or background contributions (blue dashed curves), which for the valence band is in line with the results of Ref. 38. The overall agreement between the calculated photocurrent and experiment is reasonably good. The distance of the satellites from the quasiparticle position is systematically slightly overestimated; this may be due to the use of the RPA that overestimates plasmon frequencies in loss or inelastic x-ray scattering spectra, especially with increasing momentum transfer. ${ }^{63,64}$ Since the screening of $W$ should correspond to the measurable one in order to obtain the correct spectrum, this leads to an error in the satellite positions. This discrepancy in peak position also partly accounts for the discrepancy in satellite amplitude due to the $1 / \omega^{2}$ factor in the cumulant. Note that the disagreement is larger for the valence spectrum than for the core. This is partly due to the fact that the imaginary part of the self-energy is broader and more asymmetric for states at the Fermi surface than for the core or even the valence at the $\Gamma$ point, and these states near the Fermi surface make up the dominant contribution to the valence spectrum. Since the time-ordered cumulant approach should work best far from the Fermi level, it is not astonishing that the largest deviations are found close to the Fermi level. Moreover, our model of the extrinsic and interference effects assumes that the total excitation spectrum is proportional to the intrinsic excitation spectrum, while in reality, the extrinsic effects should be roughly independent of hole level. A more appropriate way to model the extrinsic and interference terms might be to calculate these terms directly from the loss function. Also note that the position of the core levels has been aligned to zero; otherwise, the GWA $2 \mathrm{~s}$ level is too deep by about $5 \mathrm{eV}$, and the $2 \mathrm{p}$ level by about $3 \mathrm{eV}$. However, all of the main trends are reproduced by the cumulant + extrinsic + interference approach: the appearance of a satellite series, the fact that the relative weight of the satellites is larger in the valence than in the core region, and the extended incoherent spectrum that can be seen in the figures for the core levels. 

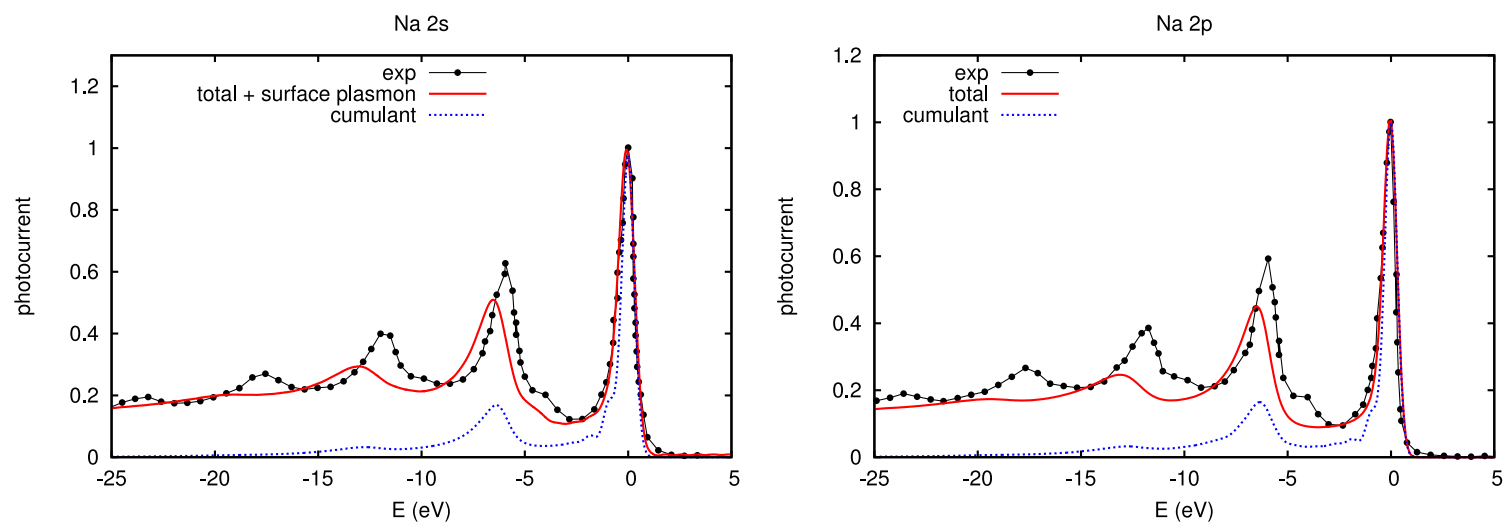

FIG. 5. Comparison of the experimental photoemission spectrum to the spectrum calculated using the cumulant + extrinsic + interference approach: left, Na $2 \mathrm{~s}$; right: $\mathrm{Na} 2 \mathrm{p}$. The red solid lines are total calculations, including secondary electron background, black lines with circles are experimental data, ${ }^{62}$ and blue dashed lines are calculated cumulant spectral functions. The total $\mathrm{Na} 2 \mathrm{~s}$ calculated spectrum also includes an approximation to the surface plasmon contribution.

\section{A UNIFIED VIEW: COUPLING OF EXCITATIONS IN THE TWO-PARTICLE GREEN'S FUNCTION}

\section{A. Dynamical effects in the two-particle Green's function}

The dynamical effects in the one-body Green's function express the fact that electron addition or removal induces excitations in the system of all electrons. In the same way, the addition of two electrons or two holes, or the excitation of an electron-hole pair, perturbs the system and should lead to additional excitations that renormalize energies, change intensities, and can lead to additional structure in the spectra. Indeed, this is an important fact that manifests itself, for example, as the so-called double excitations in molecules, ${ }^{65,66}$ or as satellites that appear at large momentum transfer in the inelastic $\mathrm{x}$ ray scattering spectra of simple metals. ${ }^{67,68}$ It is also tightly linked to the problem of extrinsic and interference effects in photoemission: the need for those corrections is due to the fact that one approximates the photoemission process in a threestep picture by the creation of a hole (the intrinsic spectral function) and the subsequent propagation of the electron to the detector (leading to extrinsic effects). A unified description should treat this as a two-particle problem, given by the hole and the electron. Both electron and hole are described by onebody Green's functions with their respective dynamical effects as outlined above, and moreover, there is in general an electronhole interaction. Such a problem can be described, for example, by the Bethe-Salpeter equation (BSE) for the two-particle correlation function $L$. In the standard approximation to the BSE, the electron-hole interaction is approximately derived from the GWA. The result is given by the screened Coulomb interaction $W$ in a static approximation. ${ }^{10}$ However, in principle, $W$ is frequency dependent: this frequency dependent $W$ leads to the interference effects, as also discussed in the following.

Solving the BSE with full frequency dependence is a very difficult problem, and several approximations have been proposed. ${ }^{69-74}$ One can however doubt whether an approximation based on the GWA would be a good starting point for the description of dynamical effects since, as we have seen above, the GWA often fails to describe satellites. It is therefore desirable to sketch a route that follows the lines of the cumulant approximation for the one-body Green's function. This is the topic of the present explorative section.

\section{B. A functional differential equation for the two-particle correlation function}

The first task is to derive a functional differential equation for the quantity of interest. Here, we will concentrate on the irreducible two-particle correlation function $\tilde{L}$, from which the reducible $L$ and spectra can be obtained straightforwardly. In analogy to (4), the irreducible two-particle correlation function is defined as

$$
\frac{\delta G_{u}\left(2,1^{\prime}\right)}{\delta u_{\mathrm{cl}}(3)}=\tilde{L}_{u}\left(2,3,1^{\prime}, 3^{+}\right),
$$

with $u_{\mathrm{cl}}$ given in (6). We now rewrite (7) as

$$
G_{u}(1,2)=G_{H u}(1,2)+i G_{H u}(1, \overline{3}) W(\overline{3}, \overline{4}) \tilde{L}_{u}\left(\overline{3}, \overline{4}^{+}, 2, \overline{4}^{++}\right)
$$

keeping $W_{u} \rightarrow W(u=0)$ fixed in the spirit of the linear response approximation. Here, we have defined $G_{H u}(1,2)$ $=G^{0}(1,2)+G^{0}(1, \overline{3}) u_{\mathrm{cl}}(\overline{3}) G_{H u}(\overline{3}, 2)$. Differentiation with respect to $u_{\mathrm{cl}}$, using the fact that the last term contains a double derivative of $G$, yields a functional differential equation for $\tilde{L}_{u}$,

$$
\begin{aligned}
\tilde{L}_{u}\left(1,5,2,5^{+}\right)= & G_{H u}(1,5) G_{H u}\left(5^{+}, 2\right) \\
& +i G_{H u}(1,5) G_{H u}\left(5^{+}, \overline{3}\right) W(\overline{3}, \overline{4}) \tilde{L}_{u}\left(\overline{3}, \overline{4}^{+}, 2, \overline{4}^{++}\right) \\
& +i G_{H u}(1, \overline{3}) W(\overline{3}, \overline{4}) \frac{\delta \tilde{L}_{u}\left(\overline{3}, 5,2,5^{+}\right)}{\delta u_{\mathrm{cl}}(\overline{4})}
\end{aligned}
$$

Substitution of (29) finally leads to

$$
\begin{aligned}
\tilde{L}_{u}\left(1,5,2, \overline{5}^{+}\right)= & G_{H u}(1,5) G_{u}\left(5^{+}, 2\right) \\
& +i G_{H u}(1, \overline{3}) W(\overline{3}, \overline{4}) \frac{\delta \tilde{L}_{u}\left(\overline{3}, 5,2,5^{+}\right)}{\delta u_{\mathrm{cl}}(\overline{4})} .
\end{aligned}
$$

This is a functional differential equation for $\tilde{L}_{u}$ in terms of $u_{\mathrm{cl}}$, provided $G_{u}$ is given. It is now most natural to use the results of above, namely, to write the equation in a basis, make the decoupling approximation, and use the cumulant solution for $G_{u}$. In this way, one obtains a differential equation for one transition matrix element $\mathcal{L}_{v c} \equiv \tilde{L}_{u}^{v v c c}$, for a resonant transition $v \rightarrow c$ from a valence to a conduction state. The differential 
equation reads

$$
\begin{aligned}
\mathcal{L}_{v c}\left(t_{1}, t_{5}, t_{2}, t_{5}^{+}\right)= & \mathcal{G}_{v v}^{H u}\left(t_{1}, t_{5}\right) \mathcal{G}_{c c}^{u}\left(t_{5}^{+}, t_{2}\right) \\
& +i \int_{t_{1}}^{t_{5}} \int_{-\infty}^{+\infty} \sum_{n=v, c} \mathcal{G}_{v v}^{H u}\left(t_{1}, t_{\overline{3}}\right) W_{v v n n}\left(t_{\overline{3}}, t_{\overline{4}}\right) \\
& \times \frac{\delta \mathcal{L}_{v c}\left(t_{\overline{3}}, t_{5}, t_{2}, t_{5}^{+}\right)}{\delta u_{\mathrm{cl}, n n}\left(t_{\overline{4}}\right)} d t_{\overline{3}} d t_{\overline{4}}
\end{aligned}
$$

In the unusual zero-order term $\mathcal{G}^{H u} \mathcal{G}^{u}$, the hole interacts via the Hartree potential with the rest of the system, while the electron propagates with a fully interacting Green's function. This asymmetric treatment makes an exponential of $W_{c c c c}$ appear (carried by the cumulant $\mathcal{G}^{u}$ ) while no equivalent term is carried by $\mathcal{G}^{H u}$. However, the missing single-particle $W_{v v v v}$ and the interaction term $W_{v v c c}$ are actually included by the sum over the index $n$ of Eq. (32).
If one starts to iterate this equation, it becomes clear that in the last term a factor

$$
\mathcal{G}_{v v}^{H u}\left(t_{1}, t_{\overline{3}}\right) \mathcal{G}_{v v}^{H u}\left(t_{\overline{3}}, t_{5}\right) \mathcal{G}^{u}\left(t_{5}, t_{2}\right) \mathcal{F}_{v c}[W]
$$

appears at any order, where $\mathcal{F}_{v c}[W]$ is a functional of $W$ but not of $u_{\mathrm{cl}}$. Moreover, $\mathcal{G}_{v v}^{H u}\left(t_{1}, t_{\overline{3}}\right) \mathcal{G}_{v v}^{H u}\left(t_{\overline{3}}, t_{5}\right)=\mathcal{G}_{v v}^{H u}\left(t_{1}, t_{5}\right)$. The full equation is then solved by means of the ansatz $\tilde{\mathcal{L}}_{v c}=\mathcal{G}_{v v}^{H u}$ $\mathcal{G}_{c c}^{u} \mathcal{F}_{v c}$, suggested by the result of the iteration procedure.

The solution for $\mathcal{F}$ follows the same scheme as for the single particle Green's function, and it leads to two exponentials of $W_{v v c c}$ and $W_{v v v v}$ analogous to the exponential term in the cumulant. The equilibrium limit $u=0$ can finally be taken.

\section{Solution and discussion}

For $u=0$, the solution of Equation (32) is

$$
\begin{aligned}
\mathcal{L}_{v c}\left(t_{1}, t_{5}, t_{2}, t_{5}^{+}\right) & =e^{-i\left[\varepsilon_{c}^{H}\left(t_{5}-t_{2}\right)-\varepsilon_{v}^{H}\left(t_{5}-t_{1}\right)\right]} e^{-\frac{i}{2} \int_{t_{2}}^{t_{5}} \int_{t_{2}}^{t_{5}} W_{c c c c}\left(t-t^{\prime}\right) d t d t^{\prime}-\frac{i}{2} \int_{t_{1}}^{t_{5}} \int_{t_{1}}^{t_{5}} W_{v v v v}\left(t-t^{\prime}\right) d t d t^{\prime}+i \int_{t_{1}}^{t_{5}} \int_{t_{2}}^{t_{5}} W_{v v c c}\left(t-t^{\prime}\right) d t d t^{\prime}} \\
& =\mathcal{G}_{v v}\left(t_{1}, t_{5}\right) \mathcal{G}_{c c}\left(t_{5}, t_{2}\right) e^{i \int_{t_{1}}^{t_{5}} \int_{t_{2}}^{t_{5}} W_{v v c c}\left(t-t^{\prime}\right) d t d t^{\prime}}
\end{aligned}
$$

The polarizability (limit $t_{2} \rightarrow t_{1}$ ) can be taken only at the end of the calculation, once the exponential solution is found and the vanishing $u$ limit can be performed.

Now, we have a cumulant with three contributions: the quasi-particle correction and excitations due to the hole in $W_{v v v v}$ (the original intrinsic contribution in the one-body Green's function), quasi-particle corrections and excitations due to the electron in $W_{c c c c}$, which give rise to the extrinsic effects, and the mixed term $W_{v v c c}$ that is responsible for excitonic effects and for the interference effects in the context of photoemission. Note that when all matrix elements are equal, there is perfect cancellation because of the opposite signs and factors $1 / 2$. This provides a justification for the complete neglect of dynamical effects that is usually done in Bethe-Salpeter calculations. The result shows that it would be dangerous to neglect only part of the effects, for example, include only the dynamical effects in the electron-hole interaction via $W_{v v c c}$ but keep the quasi-particle approximations for the one-body Green's functions. Such a cancellation of dynamical effects has been discussed in Ref. 75. In that work, only the first order expression was derived, but mixing of transitions at different k-points was allowed. It should be clear that this mixing is crucial if one wants to use the results for calculations in extended systems, since excitonic effects in these systems can only occur when transitions are allowed to mix: in the thermodynamic limit, the first-order correction to the energies vanishes. Our derivation above has to be understood as a basis for discussion, and as a first step towards the calculation of spectra including dynamical effects in the two-body Green's function to all order, along the lines that have shown to be very successful for the one-body Green's function.

\section{CONCLUSIONS}

In conclusion, dynamical effects in electronic spectra are a signature of correlation, since they cannot be explained in any independent-particle picture. They express the coupling of electrons to different excitations in the system. In the case of photoemission, the coupling of a hole to electron-hole pairs and plasmons can efficiently be described by extending the framework of the GW approximation to include multiple satellites via a cumulant approach, because the screened Coulomb interaction $W$ contains this kind of excitations. The cumulant form for the one-body Green's function has been known for many years, and it is the exact solution of a boson excitation model for core-level spectroscopy. Here, we have explained how one can obtain it as an approximation on the same footing as the GWA, which highlights the main assumptions and eventually allows one to go beyond. As an example for the potential of the theoretical approach, we have sketched a way to apply the same procedure to the two-body Green's function. We have illustrated the main findings with ab initio calculations of the one-body spectral function of bulk sodium, going beyond published results by extending the study to the core region, where we show the crucial importance of self-consistency. We also add extrinsic and interference effects, and finally obtain electron removal spectra in very good agreement with experiment.

The two main approximations used here are a linearresponse treatment of the Hartree potential and an approximate way to take coupling between excitations into account. Work is under way to make the latter procedure more systematic and to understand the drawbacks of the linear-response approximation. For example, it is clear that satellites due to hole-hole 
excitations, such as the famous $6 \mathrm{eV}$ satellite in nickel, ${ }^{76-80}$ cannot be described in this way, because $W$ contains only electron-hole excitations. Nevertheless, the progress of this approach with respect to satellite spectra calculated in the GWA is striking, at a computational cost that is negligible with respect to the GWA calculation. Whether the same will be true for the two-body Green's function, where mixing of k-points is crucial in order to get excitonic effects in solids, is still to be seen.

\section{ACKNOWLEDGMENTS}

The research leading to these results has received funding from the European Research Council under the European Union's Seventh Framework Programme (No. FP/2007-2013)/ ERC Grant Agreement No. 320971 and from a Marie Curie FP7 Integration Grant. Computational time was granted by GENCI (Project No. 544). J.J.R. acknowledges hospitality by the Ecole Polytechnique, with financial support by the Labex NanoSaclay and the chaire X-ESPCI-Saint Gobain "Sciences des Matériaux et Surfaces Actives." J.J.K. and J.J.R. are also supported in part by the US DOE BES Grant No. DE-FG0397ER45623. We acknowledge fruitful discussions with Marco Cazzaniga.

${ }^{1}$ P. Hohenberg and W. Kohn, Phys. Rev. 136, B864 (1964).

${ }^{2}$ W. Kohn and L. J. Sham, Phys. Rev. 140, A1133 (1965).

${ }^{3}$ J. P. Perdew, R. G. Parr, M. Levy, and J. L. Balduz, Phys. Rev. Lett. 49, 1691 (1982).

${ }^{4}$ C.-O. Almbladh and U. von Barth, Phys. Rev. B 31, 3231 (1985).

${ }^{5}$ M. van Schilfgaarde, T. Kotani, and S. Faleev, Phys. Rev. Lett. 96, 226402 (2006).

${ }^{6}$ A. L. Fetter and J. D. Walecka, Quantum Theory of Many-Particle Systems (McGraw-Hill, New York, 1971).

${ }^{7}$ E. Economou, Green's Functions in Quantum Physics, 2nd ed. (SpringerVerlag, Berlin, 1992).

${ }^{8}$ W. G. Aulbur, L. Jonsson, and J. W. Wilkins, Solid State Phys. 54, 1 (2000).

${ }^{9}$ F. Aryasetiawan and O. Gunnarsson, Rep. Prog. Phys. 61, 237 (1998).

${ }^{10}$ G. Onida, L. Reining, and A. Rubio, Rev. Mod. Phys. 74, 601 (2002).

${ }^{11}$ T. Koopmans, Physica 1, 104 (1934).

${ }^{12}$ L. Hedin, Phys. Rev. 139, A796 (1965).

${ }^{13}$ D. Bohm and D. Pines, Phys. Rev. 92, 609 (1953).

${ }^{14}$ G. Strinati, H. J. Mattausch, and W. Hanke, Phys. Rev. B 25, 2867 (1982).

${ }^{15}$ M. S. Hybertsen and S. G. Louie, Phys. Rev. Lett. 55, 1418 (1985).

${ }^{16}$ F. Bruneval, N. Vast, L. Reining, M. Izquierdo, F. Sirotti, and N. Barrett, Phys. Rev. Lett. 97, 267601 (2006).

${ }^{17}$ M. Gatti, F. Bruneval, V. Olevano, and L. Reining, Phys. Rev. Lett. 99, 266402 (2007).

${ }^{18}$ S. V. Faleev, M. van Schilfgaarde, and T. Kotani, Phys. Rev. Lett. 93, 126406 (2004).

${ }^{19}$ F. Bruneval and M. Gatti, in First Principles Approaches to Spectroscopic Properties of Complex Materials, Topics in Current Chemistry Vol. 347, edited by C. Di Valentin, S. Botti, and M. Cococcioni (Springer, Berlin Heidelberg, 2014), pp. 99-135, ISBN: 978-3-642-55067-6.

${ }^{20}$ M. Guzzo, G. Lani, F. Sottile, P. Romaniello, M. Gatti, J. J. Kas, J. J. Rehr, M. G. Silly, F. Sirotti, and L. Reining, Phys. Rev. Lett. 107, 166401 (2011).

${ }^{21}$ T. Åberg, Phys. Rev. 156, 35 (1967).

${ }^{22}$ A. D. O. Bawagan and E. R. Davidson, Adv. Chem. Phys. 110, 215 (1999).

${ }^{23}$ A. G. Marinopoulos, L. Reining, V. Olevano, A. Rubio, T. Pichler, X. Liu, M. Knupfer, and J. Fink, Phys. Rev. Lett. 89, 266406 (2002).

${ }^{24}$ R. Hambach, C. Giorgetti, N. Hiraoka, Y. Q. Cai, F. Sottile, A. G. Marinopoulos, F. Bechstedt, and L. Reining, Phys. Rev. Lett. 101, 266406 (2008).

${ }^{25}$ C. Kramberger, R. Hambach, C. Giorgetti, M. H. Rümmeli, M. Knupfer, J. Fink, B. Büchner, L. Reining, E. Einarsson, S. Maruyama et al., Phys. Rev. Lett. 100, 196803 (2008).
${ }^{26}$ S. Waidmann, M. Knupfer, B. Arnold, J. Fink, A. Fleszar, and W. Hanke, Phys. Rev. B 61, 10149 (2000).

${ }^{27}$ H. Dröge, A. Fleszar, W. Hanke, M. Sing, M. Knupfer, J. Fink, F. Goschenhofer, C. R. Becker, R. Kargerbauer, and H. P. Steinrück, Phys. Rev. B 59, 5544 (1999).

${ }^{28}$ J. Schwinger, Proc. Natl. Acad. Sci. U. S. A. 37, 452 (1951).

${ }^{29}$ G. Lani, P. Romaniello, and L. Reining, New J. Phys. 14, 013056 (2012).

${ }^{30}$ L. P. Kadanoff and G. Baym, Quantum Statistical Mechanics (W. A. Benjamin, Inc., New York, 1964).

${ }^{31}$ M. Gatti and M. Guzzo, Phys. Rev. B 87, 155147 (2013).

${ }^{32}$ D. C. Langreth, Phys. Rev. B 1, 471 (1970).

${ }^{33}$ P. Noziéres and C. T. De Dominicis, Phys. Rev. 178, 1097 (1969)

${ }^{34}$ F. Bechstedt, Phys. Status Solidi B 101, 275 (1980).

${ }^{35}$ F. Bechstedt, R. Enderlein, and M. Koch, Phys. Status Solidi B 99, 61 (1980).

${ }^{36}$ F. Bechstedt, Phys. Status Solidi B 112, 9 (1982).

${ }^{37}$ C.-O. Almbladh and L. Hedin, in Handbook on Synchrotron Radiation (North-Holland, Amsterdam, 1983), Chap. 8.

${ }^{38}$ F. Aryasetiawan, L. Hedin, and K. Karlsson, Phys. Rev. Lett. 77, 2268 (1996).

${ }^{39}$ L. Hedin, Phys. Scr. 21, 477 (1980).

${ }^{40}$ A. S. Kheifets, V. A. Sashin, M. Vos, E. Weigold, and F. Aryasetiawan, Phys. Rev. B 68, 233205 (2003).

${ }^{41}$ M. Vos, A. Kheifets, V. Sashin, E. Weigold, M. Usuda, and F. Aryasetiawan, Phys. Rev. B 66, 155414 (2002).

${ }^{42}$ J. Lischner, D. Vigil-Fowler, and S. G. Louie, Phys. Rev. B 89, 125430 (2014).

${ }^{43}$ F. Caruso, H. Lambert, and F. Giustino, Phys. Rev. Lett. 114, 146404 (2015).

${ }^{44}$ J. Lischner, G. K. Pálsson, D. Vigil-Fowler, S. Nemsak, J. Avila, M. C. Asensio, C. S. Fadley, and S. G. Louie, Phys. Rev. B 91, 205113 (2015).

${ }^{45}$ M. Guzzo, J. Kas, F. Sottile, M. Silly, F. Sirotti, J. Rehr, and L. Reining, Eur. Phys. J. B 85, 324 (2012).

${ }^{46}$ M. Guzzo, J. J. Kas, L. Sponza, C. Giorgetti, F. Sottile, D. Pierucci, M. G. Silly, F. Sirotti, J. J. Rehr, and L. Reining, Phys. Rev. B 89, 085425 (2014).

${ }^{47}$ J. J. Kas, J. J. Rehr, and L. Reining, Phys. Rev. B 90, 085112 (2014).

${ }^{48}$ J. J. Kas, A. P. Sorini, M. P. Prange, L. W. Cambell, J. A. Soininen, and J. J. Rehr, Phys. Rev. B 76, 195116 (2007).

${ }^{49}$ X. Gonze, G. M. Rignanese, M. Verstraete, J. M. Beuken, Y. Pouillon, R. Caracas, F. Jollet, M. Torrent, G. Zerah, M. Mikami et al., Z. Kristallogr. 220, 558 (2005).

${ }^{50}$ M. Cazzaniga, Phys. Rev. B 86, 035120 (2012).

${ }^{51}$ R. Wyckoff, Crystal Structures, 2nd ed. (Interscience Publishers, New York, 1963), Vol. 1.

${ }^{52}$ N. Troullier and J. L. Martins, Phys. Rev. B 43, 1993 (1991).

${ }^{53}$ M. Gatti, I. V. Tokatly, and A. Rubio, Phys. Rev. Lett. 104, 216404 (2010).

${ }^{54}$ T. J. Pollehn, A. Schindlmayr, and R. W. Godby, J. Phys.: Condens. Matter 10, 1273 (1998).

${ }^{55}$ M. Gatti, G. Panaccione, and L. Reining, Phys. Rev. Lett. 114, 116402 (2015).

${ }^{56}$ A. Damascelli, Z. Hussain, and Z.-X. Shen, Rev. Mod. Phys. 75, 473 (2003).

${ }^{57}$ W. Bardyszewski and L. Hedin, Phys. Scr. 32, 439 (1985).

${ }^{58}$ L. Hedin, J. Michiels, and J. Inglesfield, Phys. Rev. B 58, 15565 (1998).

${ }^{59}$ J. E. Inglesfield, J. Phys. C 16, 403 (1983).

${ }^{60}$ J. B. Pendry, Low Energy Electron Diffraction (Academic Press, London, 1974).

${ }^{61}$ H. Höchst, P. Steiner, and S. Hüfner, Z. Phys. B: Condens. Matter 30, 145 (1978).

${ }^{62}$ P. Steiner, H. Höchst, and S. Hüfner, Z. Phys. B: Condens. Matter 30, 129 (1978).

${ }^{63}$ M. Cazzaniga, H.-C. Weissker, S. Huotari, T. Pylkkänen, P. Salvestrini, G. Monaco, G. Onida, and L. Reining, Phys. Rev. B 84, 075109 (2011).

${ }^{64}$ S. Huotari, M. Cazzaniga, H.-C. Weissker, T. Pylkkänen, H. Müller, L. Reining, G. Onida, and G. Monaco, Phys. Rev. B 84, 075108 (2011).

${ }^{65}$ P. Romaniello, D. Sangalli, J. A. Berger, F. Sottile, L. G. Molinari, L. Reining, and G. Onida, J. Chem. Phys. 130, 044108 (2009).

${ }^{66}$ D. Sangalli, P. Romaniello, G. Onida, and A. Marini, J. Chem. Phys. 134, 034115 (2011).

${ }^{67}$ C. Sternemann, S. Huotari, G. Vankó, M. Volmer, G. Monaco, A. Gusarov, H. Lustfeld, K. Sturm, and W. Schülke, Phys. Rev. Lett. 95, 157401 (2005). 
${ }^{68}$ S. Huotari, C. Sternemann, W. Schülke, K. Sturm, H. Lustfeld, H. Sternemann, M. Volmer, A. Gusarov, H. Müller, and G. Monaco, Phys. Rev. B 77, 195125 (2008).

${ }^{69}$ K. Shindo, J. Phys. Soc. Jpn. 29, 287 (1970).

${ }^{70}$ R. Zimmermann, K. Kilimann, W. D. Kraeft, D. Kremp, and G. Ropke, Phys. Status Solidi B 90, 175 (1978).

${ }^{71}$ G. Strinati, Phys. Rev. Lett. 49, 1519 (1982).

${ }^{72}$ G. Strinati, Phys. Rev. B 29, 5718 (1984).

${ }^{73}$ M. Rohlfing and S. G. Louie, Phys. Rev. B 62, 4927 (2000).
${ }^{74}$ A. Marini and R. Del Sole, Phys. Rev. Lett. 91, 176402 (2003).

${ }^{75}$ F. Bechstedt, K. Tenelsen, B. Adolph, and R. Del Sole, Phys. Rev. Lett. 78, 1528 (1997).

${ }^{76}$ D. R. Penn, Phys. Rev. Lett. 42, 921 (1979).

${ }^{77}$ A. Liebsch, Phys. Rev. Lett. 43, 1431 (1979).

${ }^{78}$ A. Liebsch, Phys. Rev. B 23, 5203 (1981).

${ }^{79}$ M. Cini, Surf. Sci. 87, 483 (1979).

${ }^{80}$ M. Springer, F. Aryasetiawan, and K. Karlsson, Phys. Rev. Lett. 80, 2389 (1998). 